\title{
Some Special Features of a Rotating-Moving $\mathbf{D} p$-Brane
}

\author{
Farzin Safarzadeh-Maleki \\ f.safarzadeh@aut.ac.ir
}

\begin{abstract}
Using the boundary string field theory (BSFT) techniques we study the boundary state and partition function for a dynamical (rotating-moving) $\mathrm{D} p$-brane coupled to the electromagnetic and tachyonic background fields in superstring theory. By making use of the created partition function, the super BSFT action with a tachyonic field and Dirac-Born-Infeld type action will be constructed. By analyzing the obtained action interesting features will be revealed.
\end{abstract}

PACS numbers: 11.25.-w; 11.25.Uv

Keywords: Rotating-Moving brane; Boundary state; Partition function; BSFT; DBI.

\section{Introduction}

Many aspects of string/superstring theory have been discovered by studying the essential objects of the theory name as D-branes $[1,2]$. By means of the boundary state method [3, 4], several properties and different configurations of D-branes have been investigated [5, 6]. The low-energy dynamics of a D-brane can be described by an effective action, i.e., Dirac-BornInfeld (DBI), plus its coupling to the Ramond-Ramond fields. A DBI action is the unique invariant term under the broken Lorentz symmetry in the lowest approximation. It has the reparameterization invariance which removes the longitudinal fluctuations of the brane and also is invariant under the full space time symmetry [7].

On the other hand, in the context of background independent open string field theory or BSFT for the case of superstring theory, an effective spacetime action can be extracted by the corresponding world sheet partition function. Moreover, these concepts have been related to the boundary state formalism in an elegant way [8]-[10].

In this article by means of the BSFT techniques, we explore the boundary state and partition function for a rotating-moving $\mathrm{D} p$-brane coupled to a $U(1)$ gauge potential in the world volume of the brane and tachyonic background field in superstring theory. Then by making use of the created partition function, we study the effective action of such a system. In other words, we derive the super BSFT space time action with a tachyonic field and DBItype photonic field associated with a rotating-moving brane from the world sheet action. 
The existence of the tachyonic boundary interaction breaks conformal invariance and makes the theory off-shell. Therefore, the BSFT would be a proper formalism to study such a dynamical system. Being a relevant interaction, it will induce an RG flow between two conformal field theories which describes tachyon condensation $[9,11]$.

The generalized BSFT action corresponding to this rotating-moving $\mathrm{D} p$-brane with background fields, enables us to gain a new understanding of this dynamical system properties. Through analyzing the action, among other interesting specifications such as existence of a mixing structure between the tachyonic field and rotating-moving term which affects the dynamics of the action, by eliminating the effect of the zero mode portion of the partition function an outstanding feature shows up: the rotation does not generate any DBI-type factor, therefore, in spite of the fact that we start from a rotating moving world sheet brane action, we obtain the space time action without any contribution of the angular momentum and the moment of inertia. This point will be investigated both by means of the boundary state and partition function approaches. Furthermore, by some simplifications, the obtained action can be studied in two limits of small and large values of tachyonic field. The former limit could generate the right corrections of the effective action and the latter can construct the conventional tachyon effective action describing the dynamics of the tachyon field on a non-BPS Dp-brane of type IIA or IIB superstring theory.

This article is organized as follows. In Sec. 2, the world sheet action of a rotatingmoving $\mathrm{D} p$-brane with various background fields in bosonic and superstring theory will be constructed. In Sec. 3, The boundary state of such a dynamical will be extracted. In Sec. 4, The partition function and DBI-type action associated with a rotating-moving brane will be investigated.

\section{The actions}

In the context of BSFT or from the boundary sigma model point of view for the closed string, in order to construct a dynamical (rotating-moving) D-brane system in the presence of various fields, we start with a bulk action

$$
S_{\mathrm{bulk}}=-\frac{1}{4 \pi \alpha^{\prime}} \int_{\Sigma} d^{2} \sigma\left(\sqrt{-g} g^{a b} G_{\mu \nu} \partial_{a} X^{\mu} \partial_{b} X^{\nu}\right)
$$

and then couple the deformations to the original theory via the boundary terms, as follows

$$
S_{\mathrm{bdry}}=\frac{1}{2 \pi \alpha^{\prime}} \int_{\partial \Sigma} d \sigma\left(A_{\alpha} \partial_{\sigma} X^{\alpha}+\omega_{\alpha \beta} J_{\tau}^{\alpha \beta}+i T^{2}\left(X^{\alpha}\right)\right)
$$


Therefore the desired action would be $S^{\text {Bosonic }}=S_{\text {bulk }}+S_{\text {bdry }}$, in which $\Sigma$ is the closed string world-sheet, exchanged between the branes, and $\partial \Sigma$ is its boundary which is a world sheet space-like surface. This action contains a $U(1)$ gauge field $A_{\alpha}$ which lives in the worldvolume of the brane, an $\omega$-term associated with the rotation and motion of the brane and a tachyonic field. We shall use $\left\{X^{\alpha} \mid \alpha=0,1, \cdots, p\right\}$ for the world-volume directions of the brane and $\left\{X^{i} \mid i=p+1, \cdots, d-1\right\}$ for directions perpendicular to it.

For simplifying the calculations the background field $G_{\mu \nu}$ is considered to be constant. In addition, for the $U(1)$ gauge field we apply the gauge $A_{\alpha}=-\frac{1}{2} F_{\alpha \beta} X^{\beta}$ with constant field strength. Besides, we use the following tachyon profile $T^{2}=T_{0}+\frac{1}{2} U_{\alpha \beta} X^{\alpha} X^{\beta}$, where $T_{0}$ and the symmetric matrix $U_{\alpha \beta}$ are constant. Finally, the $\omega$-term, which is responsible for the brane's rotation and motion, contains the anti-symmetric angular velocity $\omega_{\alpha \beta}$ and angular momentum density $J_{\tau}^{\alpha \beta}$ which is given by $\omega_{\alpha \beta} J_{\tau}^{\alpha \beta}=2 \omega_{\alpha \beta} X^{\alpha} \partial_{\tau} X^{\beta}$. In fact, the component $\left.\omega_{0 \bar{\alpha}}\right|_{\bar{\alpha} \neq 0}$ denotes the velocity of the brane along the direction $X^{\bar{\alpha}}$ while $\omega_{\bar{\alpha} \bar{\beta}}$ represents its rotation. Note that the rotation of the brane is considered to be in its volume and its motion along the brane directions. Moreover, according to the various fields inside the brane, the Lorentz symmetry is broken and hence such a dynamic is meaningful.

The purpose of choosing the constant field strength and such a tachyon profile is that for sufficiently small values, an expansion of regularized action gives the right corrections and one can study the influence of these couplings on the effective action. On the other hand, because of losing the conformal invariance, due to the presence of tachyon field, the influence of these fields has been entered as corrections to the effective action. The importance of this statement will be revealed in Sec. 4.

In order to supersymmetrize the above action one should add fermionic partners of the $X^{\mu}$ 's , i.e., $\psi^{\mu}$ 's such that the supersymmetrized action would be invariant under the global worldsheet supersymmetry. Steps of supersymmetrizing the bosonic action using a superspace formalism can be summarized as: first, using the super world-sheet coordinates and hence, adding the Grassmanian integration measurement; second, changing the ordinary derivative to the super covariant derivative and third, changing the fields to the superfields which are the quantum mechanical degrees of freedom living on the boundary and the restrictions to the boundary of the standard world sheet super coordinate $[1,8,12]$. Therefore, the supersymmetrized version of the rotating-moving world sheet action with photonic and tachyonic background fields would be $S_{\text {bdry+bulk }}^{\text {Super }}=S^{\text {Fermionic }}+S^{\text {Bosonic }}$, in which

$$
S^{\text {Fermionic }}=\frac{-i}{4 \pi \alpha^{\prime}} \int_{\partial \Sigma} d \sigma\left[\theta^{\alpha} F_{\alpha \beta} \theta^{\beta}+4 \omega_{\alpha \beta}\left[\left(\psi_{+}^{\alpha}+i \eta \psi_{-}^{\alpha}\right)\left(\psi_{+}^{\beta}-i \eta \psi_{-}^{\beta}\right)\right]+i\left(\theta^{\alpha} \partial_{\alpha} T\right) \partial_{\sigma}^{-1}\left(\theta^{\beta} \partial_{\beta} T\right)\right]
$$




$$
+\frac{i}{4 \pi \alpha^{\prime}} \int_{\Sigma} d^{2} \sigma\left(\bar{\psi}^{\mu} \rho^{a} \partial_{a} \psi^{\mu}\right)
$$

where the boundary fermion $\theta^{\alpha}=\psi_{+}^{\alpha}+i \eta \psi_{-}^{\alpha}$ is the linear combination of the solution of the closed superstring equations of motion $\psi^{\alpha}=\left(\begin{array}{c}\psi_{+}^{\alpha} \\ \psi_{-}^{\alpha}\end{array}\right)$. Moreover, $\partial_{\sigma}^{-1}$ is a Green function that could be written in terms of the sign function $\partial_{\sigma}^{-1} g(\sigma)=\frac{1}{2} \int d \sigma^{\prime} \operatorname{sgn}\left(\sigma-\sigma^{\prime}\right) \mathrm{g}\left(\sigma^{\prime}\right)$ and $\rho^{a}$ 's are two dimensional Dirac matrices. We use the same notation as [10].

\section{Boundary state of the system}

By means of the obtained action, one can define the associated bosonic boundary state as

$$
\begin{aligned}
& \left|B ; S_{\text {Bos }}^{\text {bdry }}\right\rangle=\int[d x d \bar{x}] e^{i S_{\text {Bos }}^{\text {bdry }}[x, \bar{x}]}|x, \bar{x}\rangle \\
& |x, \bar{x}\rangle=\prod_{m=1}^{\infty} \exp \left(-\frac{1}{2} \bar{x}_{m} x_{m}-a_{m}^{\dagger} \tilde{a}_{m}^{\dagger}+a_{m}^{\dagger} x_{m}+\bar{x}_{m} \tilde{a}_{m}^{\dagger}\right)|v a c\rangle,
\end{aligned}
$$

where $x_{m}=a_{m} e^{-2 i m \tau}+\tilde{a}_{m}^{\dagger} e^{2 i m \tau}$, defined in terms of the bosonic oscillators, can be derived from the solution of closed superstring equations of motion

$$
X^{\mu}(\sigma, \tau)=x_{0}^{\mu}+2 \alpha^{\prime} p^{\mu} \tau+\sqrt{\frac{\alpha^{\prime}}{2}} \sum_{m>0} m^{-\frac{1}{2}}\left(x_{m}^{\mu} e^{2 i m \sigma}+\bar{x}_{m}^{\mu} e^{-2 i m \sigma}\right) .
$$

Beside, the bosonic boundary action $S_{\mathrm{Bos}}^{\mathrm{bdry}}=\left(S^{\mathrm{F}}+S^{\mathrm{T}}+S^{\omega}\right)_{\text {Bos }}$ is related to the photon, tachyon and the rotation terms as follows

$$
\begin{aligned}
& S_{\mathrm{Bos}}^{\mathrm{F}}=\frac{i}{2} \sum_{m>0} F_{\alpha \beta} \bar{x}_{m}^{\alpha} x_{m}^{\beta}, \\
& S_{\mathrm{Bos}}^{\mathrm{T}}=\frac{i}{4 \alpha^{\prime}} U_{\alpha \beta} x_{0}^{\alpha} x_{0}^{\beta}+\frac{i}{4} \sum_{m>0} \frac{U_{\alpha \beta}}{m} \bar{x}_{m}^{\alpha} x_{m}^{\beta}, \\
& S_{\text {Bos }}^{\omega}=2 \omega_{\alpha \beta} x_{0}^{\alpha} p^{\beta}-2 i \omega_{\alpha \beta} \sum_{m>0}\left(\bar{x}_{m}^{\alpha} a_{m}^{\beta}+x_{m}^{\alpha} \tilde{a}_{m}^{\beta}\right) .
\end{aligned}
$$

The above equations are obtained by applying the oscillating modes in the bosonic sector. Thus by inserting the obtained $S_{\mathrm{Bos}}^{\text {bdry }}$ in Eq. (4) the solution of the bosonic part would be extracted as

$$
\begin{aligned}
\left|B ; S_{\text {Bos }}^{\text {bdry }}\right\rangle & =\frac{T_{p}}{2} \prod_{n=1}^{\infty}\left[\operatorname{det} Q_{(n)}\right]^{-1} \exp \left[-\sum_{m=1}^{\infty} \frac{1}{m} \alpha_{-m}^{\mu} S_{(m) \mu \nu} \widetilde{\alpha}_{-m}^{\nu}\right]|0\rangle_{\alpha} \otimes|0\rangle_{\widetilde{\alpha}} \\
& \times \int_{-\infty}^{\infty} \exp \left\{\alpha^{\prime}\left[\sum_{\alpha=0}^{p}\left(U^{-1} \mathbf{A}\right)_{\alpha \alpha}\left(p^{\alpha}\right)^{2}+\sum_{\alpha, \beta=0, \alpha \neq \beta}^{p}\left(U^{-1} \mathbf{A}+\mathbf{A}^{T} U^{-1}\right)_{\alpha \beta} p^{\alpha} p^{\beta}\right]\right\} \\
& \times\left(\prod_{\alpha}\left|p^{\alpha}\right\rangle d p^{\alpha}\right) \otimes \prod_{i} \delta\left(x^{i}-y^{i}\right)\left|p^{i}=0\right\rangle .
\end{aligned}
$$


in which the matrices are

$$
\begin{aligned}
& Q_{(n) \alpha \beta}=\eta_{\alpha \beta}-F_{\alpha \beta}-\frac{1}{2 n} U_{\alpha \beta}, \\
& S_{(m) \mu \nu}=\left(\Delta_{(m) \alpha \beta},-\delta_{i j}\right), \\
& \Delta_{(m) \alpha \beta}=\left(M_{(m)}^{-1} N_{(m)}\right)_{\alpha \beta}, \\
& M_{(m) \alpha \beta}=\eta_{\alpha \beta}+4 \omega_{\alpha \beta}-F_{\alpha \beta}-\frac{1}{2 m} U_{\alpha \beta}, \\
& N_{(m) \alpha \beta}=\eta_{\alpha \beta}+4 \omega_{\alpha \beta}+F_{\alpha \beta}+\frac{1}{2 m} U_{\alpha \beta}, \\
& \mathbf{A}_{\alpha \beta}=\eta_{\alpha \beta}+4 \omega_{\alpha \beta} .
\end{aligned}
$$

Similarly for the fermionic boundary state we have

$$
\begin{aligned}
& \left|B ; S_{\mathrm{Ferm}}^{\mathrm{bdry}}\right\rangle=\int[d \theta d \bar{\theta}] e^{i S_{\mathrm{Ferm}}^{\mathrm{bdr}}[\theta, \bar{\theta}]}|\theta, \bar{\theta}\rangle \\
& |\theta, \bar{\theta}\rangle=\prod_{m=1}^{\infty} \exp \left(-\frac{1}{2} \bar{\theta}_{m} \theta_{m}+i \eta \psi_{m}^{\dagger} \tilde{\psi}_{m}^{\dagger}+\psi_{m}^{\dagger} \theta_{m}-i \eta \bar{\theta}_{m} \tilde{\psi}_{m}^{\dagger}\right)|v a c\rangle,
\end{aligned}
$$

where $\theta_{m}=\tilde{\psi}_{m} e^{-2 i m \tau}-i \eta \psi_{m}^{\dagger} e^{2 i m \tau}$, described as a combination of fermionic oscillators, could be found as

$$
\theta^{\mu}=\sum_{m>0}\left(\theta_{m}^{\mu} e^{-2 i m \sigma}+\bar{\theta}_{m}^{\mu} e^{2 i m \sigma}\right)
$$

The fermionic boundary action $S_{\mathrm{Ferm}}^{\mathrm{bdry}}=\left(S^{F}+S^{T}+S^{\omega}\right)_{\text {Ferm }}$ contains

$$
\begin{aligned}
S_{\text {Ferm }}^{\mathrm{F}} & =\frac{i}{2 \alpha^{\prime}} F_{\alpha \beta} \bar{\theta}_{0}^{\alpha} \theta_{0}^{\beta}+\frac{i}{2 \alpha^{\prime}} \sum_{r>0} F_{\alpha \beta} \bar{\theta}_{r}^{\alpha} \theta_{r}^{\beta}, \\
S_{\text {Ferm }}^{\mathrm{T}} & =\frac{i}{4} \sum_{r>0} \frac{U_{\alpha \beta}}{r} \bar{\theta}_{r}^{\alpha} \theta_{r}^{\beta}, \\
S_{\text {Ferm }}^{\omega} & =2 i \omega_{\alpha \beta} \sum_{r>0}\left(\bar{\theta}_{r}^{\alpha} \tilde{\psi}_{r}^{\beta}+i \eta \psi_{r}^{\beta} \theta_{r}^{\alpha}\right) .
\end{aligned}
$$

Therefore, the NS-NS and R-R sectors possess the following fermionic boundary states

$$
\begin{aligned}
\left|B_{\mathrm{Ferm}}^{\mathrm{bdry}}\right\rangle_{\mathrm{NS}} & =\prod_{r=1 / 2}^{\infty}\left[\operatorname{det} Q_{(r)}\right] \exp \left[i \sum_{r=1 / 2}^{\infty}\left(b_{-r}^{\mu} S_{(r) \mu \nu} \widetilde{b}_{-r}^{\nu}\right)\right]|0\rangle, \\
\left|B_{\mathrm{Ferm}}^{\mathrm{bdry}}\right\rangle_{\mathrm{R}} & =\prod_{n=1}^{\infty}\left[\operatorname{det} Q_{(n)}\right] \exp \left[i \sum_{m=1}^{\infty}\left(d_{-m}^{\mu} S_{(m) \mu \nu} \widetilde{d}_{-m}^{\nu}\right)\right]|B\rangle_{\mathrm{R}}^{(0)} .
\end{aligned}
$$

The role of the explicit form of zero-mode state $|B\rangle_{\mathrm{R}}^{(0)}$ is not important here. This is because for obtaining the partition function, this state would be projected onto the bravacuum. The interested reader could find its explicit form in [6].

It should be noted that in above calculations $\alpha^{\prime}=1$ has been considered. 


\section{Partition function and DBI-type action}

It has been demonstrated that the normalization factor of the boundary state is the wellknown, DBI Lagrangian. On the other hand, by means of the partition function one can obtain the DBI-type action. This delicate relation can be described as: at the tree level the disk partition function in the BSFT appears as the normalization factor of the boundary state. The partition function can be obtained by the vacuum amplitude of the boundary state

$$
Z^{\text {disk }}=\langle\text { vacuum } \mid B\rangle
$$

Thus, in our setup the partition function possesses the following feature

$$
\begin{aligned}
Z_{\text {Bos }}^{\text {Disk }} & =\frac{T_{p}}{2} \int_{-\infty}^{\infty} \prod_{\alpha} d p^{\alpha} \exp \left\{\alpha^{\prime}\left[\sum_{\alpha=0}^{p}\left(U^{-1} \mathbf{A}\right)_{\alpha \alpha}\left(p^{\alpha}\right)^{2}+\sum_{\alpha, \beta=0, \alpha \neq \beta}^{p}\left(U^{-1} \mathbf{A}+\mathbf{A}^{T} U^{-1}\right)_{\alpha \beta} p^{\alpha} p^{\beta}\right]\right\} \\
& \times \prod_{n=1}^{\infty}\left[\operatorname{det} Q_{(n)}\right]^{-1} .
\end{aligned}
$$

for the bosonic part of the partition function, and

$$
Z_{\mathrm{Ferm}}^{\text {Disk }}=\prod_{k>0}^{\infty}\left[\operatorname{det} Q_{(k)}\right]
$$

for the fermionic part, where $k$ is half-integer (integer) for the NS-NS (R-R) sector. Considering both fermionic and bosonic parts, the total partition function in superstring theory is given by

$$
Z_{\text {total }}^{\text {Disk }}=\frac{T_{p}}{2} Z_{(0)}^{\text {Disk }} \frac{\prod_{k>0}^{\infty}\left[\operatorname{det} Q_{(k)}\right]}{\prod_{n=1}^{\infty}\left[\operatorname{det} Q_{(n)}\right]}
$$

Where

$Z_{(0)}^{\text {Disk }} \equiv \int_{-\infty}^{\infty} \prod_{\alpha} d p^{\alpha} \exp \left\{\alpha^{\prime}\left[\sum_{\alpha=0}^{p}\left(U^{-1} \mathbf{A}\right)_{\alpha \alpha}\left(p^{\alpha}\right)^{2}+\sum_{\alpha, \beta=0, \alpha \neq \beta}^{p}\left(U^{-1} \mathbf{A}+\mathbf{A}^{T} U^{-1}\right)_{\alpha \beta} p^{\alpha} p^{\beta}\right]\right\}$

In order to prevent a divergent infinite product, let us use the $\zeta$-function regularization

$$
Z_{\text {total }}^{\text {Disk }} \longrightarrow \frac{T_{p}}{2} Z_{(0)}^{\text {Disk }} \sqrt{\operatorname{det}(\eta-F)} \operatorname{det}\left[\frac{\sqrt{\pi} \Gamma\left(1-\frac{1}{2}(\eta-F)^{-1} U\right)}{\Gamma\left(\frac{1}{2}-\frac{1}{2}(\eta-F)^{-1} U\right)}\right] \text {. }
$$

where the arrow sign instead of equality has been used to show the application of this useful function.

The structure of the effective action can be formed by the interaction/boundary terms that are coupled to the world sheet action. The tree level closed string effective action can 
be studied by string partition on the sphere and in view of open string theory this action is described by the disk partition function. In superstring theory, the leading term in the latter case is called the Born-Infeld action [12]. Therefore, the extension of the DBI action or in other word, the super BSFT action with a tachyonic field and DBI-type photonic field associated with this dynamical brane would appear as

$$
S=\frac{T_{p}}{2}(\pi)^{(p+1) / 2} \int d^{p+1} \xi \frac{\sqrt{\operatorname{det}(\eta-F)}}{\sqrt{\operatorname{det}(D+H)}} \operatorname{det}\left[\frac{\sqrt{\pi} \Gamma\left(1-\frac{1}{2}(\eta-F)^{-1} U\right)}{\Gamma\left(\frac{1}{2}-\frac{1}{2}(\eta-F)^{-1} U\right)}\right],
$$

in which we have used the zero mode partition function after integrating on the momenta, where the diagonal matrix possesses the elements $D_{\alpha \beta}=\left(U^{-1} \mathbf{A}\right)_{\alpha \alpha} \delta_{\alpha \beta}$, the the matrix $H_{\alpha \beta}$ is defined by

$$
H_{\alpha \beta}= \begin{cases}\left(U^{-1} \mathbf{A}+\mathbf{A}^{T} U^{-1}\right)_{\alpha \beta}, & \alpha \neq \beta \\ 0 & , \quad \alpha=\beta\end{cases}
$$

and $\mathbf{A}$ has been introduced in Eq. (10). In addition, due to the tachyon profile we have $(\eta-F)^{-1} U=2\left[(\eta-F)^{-1}\right]^{\alpha \beta} \partial_{\alpha} T \partial_{\beta} T$, note that $T_{0}=0$ is considered for simplicity.

According to the above relations, the rotating-moving effect of the described brane can be seen in the zero mode part of the effective action (23). This is due to the fact that according to BSFT formulation for constructing a DBI Lagrangian, the oscillating part of the boundary state which contains the dynamical term, does not generate any DBI factor and hence the rotating-moving dynamic of this brane action lies in its zero mode contribution.

An interesting feature of this set up is that by applying the boundary state procedures of calculations, explicitly one can obtain that the tachyon and rotating-moving terms have a mixed structure. Using the eigenvalues obtained from the zero mode boundary state equation, one can deduce $p^{\alpha}=-\frac{1}{2}\left[(\mathbf{A})^{-1} U\right]_{\beta}^{\alpha} x^{\beta}$, which means along the world volume of the brane, momentum of the closed string depends on its center of mass position. Consequently, in the presence of the tachyon field the emitted closed string feels an exotic potential which affects its evolution. Now consider such a system without the tachyon, then by calculating the boundary state equation of the zero mode part one would obtain $2 \mathbf{A}_{\alpha \beta} p^{\beta}|B\rangle^{(0)}=0$, which shows that this case does not have a mixed structure and hence its zero mode dynamic would be independent of the background field. Therefore unlike the one with tachyonic field, with technical difficulties in calculations, a much more simple case would be appeared.

According to the non-BPS characteristic of this dynamical D-brane and its special background fields, in order to insert its contribution from the fermionic fields, one should consider its property as a $(1,0)_{p^{-}}$-system. Note that a generic $(n, m)$ could define as $(n, m)_{p}=$ 
$n|B p\rangle_{\mathrm{NS}}+m|B p\rangle_{\mathrm{R}}$, for combinations of GSO projected states on behalf of both the Neumann boundary conditions in $p+1$ and Dirichlet boundary conditions for $d-p-1$ dimensions. Thus the fermionic contribution is that of the NS-NS sector.

It has been demonstrated that the tachyon kinks, tubes, and vortices could be found by studying the equations of motion from the DBI-type effective field theory [14]. In other word, inhomogenous solutions of the equations of motion which follow from the action encode non-trivial information about the decay of higher dimensional branes into lower dimensional ones. Therefore, one can check the tachyon kinks as solutions of classical equations of motion of this rotating-moving system. Specifically, for the case in hand the spectrum of the kinks solutions becomes richer according to our special background fields.

Now let us simplify the obtained action and drop the effect of zero mode partition function $Z_{(0)}^{\text {Disk. }}$. Consequently we have

$$
S=\frac{T_{p}}{2} \int d^{p+1} \xi V(\text { Tachyonic }) \sqrt{\operatorname{det}(\eta-F)} \operatorname{det}\left[\frac{\sqrt{\pi} \Gamma\left(1-\frac{1}{2}(\eta-F)^{-1} U\right)}{\Gamma\left(\frac{1}{2}-\frac{1}{2}(\eta-F)^{-1} U\right)}\right] .
$$

Some properties of the above action are as follow:

-The kinetic part of the action is, det $\left[\frac{\sqrt{\pi} \Gamma\left(1-\frac{1}{2}(\eta-F)^{-1} U\right)}{\Gamma\left(\frac{1}{2}-\frac{1}{2}(\eta-F)^{-1} U\right)}\right]$ and the potential term is tacitly proportional to $V$ (Tachyonic) $=e^{-\frac{i}{2} T^{2}}$ due to the defined tachyon profile. It should be noted that the exact tachyon potential is obtained by setting $U_{\mu}=0$ in tachyonic profile and computing the path integral boundary action $[8,13,14]$.

- This action is a generalized form of a non-BPS D9-brane action in [8, 12] which is an interpolating action that coincide with the low-energy two-derivative effective action for a D25-brane in open bosonic string theory and the corresponding expression for the non-BPS D9-brane in type IIA theory, in the two-derivative approximation.

- In the beginning we start from a rotating-moving world sheet brane action but at the end we reach to the space time action without any contribution of the angular momentum and the moment of inertia. In other word, why does dynamic of this typical brane effective action lie in its zero mode portion? In the following two interpretations of this case are explained: The bosonic boundary state (9) consists of oscillating and zero mode parts. The first line in (9) with the infinite determinant and the exponential factor is the contribution of the oscillators which act on the vacuum of oscillators. The remaining part belongs to the zero modes, a delta function which has been included to fix the location of the D-brane by imposing an extra condition on the position operator in the transverse direction and a momentum dependent integral which comes from taking the zero mode action into account. As it seems, there is no $\delta$-function in the directions in which we perform the rotation and 
therefore the rotation acts on the zero modes. As a result, by eliminating the effect of the zero mode portion, the rotation does not generate any DBI-type factor (boundary state approach). This case is similar to that of the rotation in a delocalized Dp-brane in [5]. In other point of view, this is because of the anti-symmetric characteristic of the rotatingmoving $(\omega)$-term. As a matter of fact, this portion would be eliminated in the path integral calculations and therefore it does not have any contribution in this action (partition function approach).

- The obtained action can be checked in the limits of small and large values of tachyon field. Where the first limit could reproduce the right corrections of the effective action due to the special boundary deformation which caused the conformal invariance to be lost, and the latter that can construct the conventional tachyon effective action describing the dynamics of the tachyon field on a non-BPS D $p$-brane of type IIA or IIB superstring theory. In the following these statements would be clarified: For the first case, in the kinetic term of action (25) consider $R\left(-\frac{1}{2}(\eta-F)^{-1} U\right)=\left[\frac{\sqrt{\pi} \Gamma\left(1-\frac{1}{2}(\eta-F)^{-1} U\right)}{\Gamma\left(\frac{1}{2}-\frac{1}{2}(\eta-F)^{-1} U\right)}\right]$. Now by defining

$$
z \equiv-\frac{1}{2}(\eta-F)^{-1} U
$$

$R(z)$ could be rewritten as

$$
R(z)=\frac{4^{z} z \Gamma(z)^{2}}{2 \Gamma(2 z)}=2^{2 z-1} z B(z)
$$

in which $B(z)$ is the Beta function. One can reproduce the leading term by expanding $R(z)$ around $z=0$, hence the above action takes the following form

$$
S=\frac{T_{p}}{2} \int d^{p+1} \xi V(\text { Tachyonic }) \sqrt{\operatorname{det}(\eta-F)} \operatorname{det}\left[1+\log [4] z+\left(-\frac{\pi^{2}}{6}+2 \log [2]^{2}\right) z^{2}+O[z]^{3}\right](28
$$

It can be interpreted as the corrections to the DBI-type action according to the special format of the kinematic part of the D $p$-brane Lagrangian.

In order to construct the tachyonic effective action of a non-BPS D p-brane of type IIA or IIB superstring theory, let us check action (25) for large values of $z$. Thus, by means of the recursion relation obtained from $\Gamma(1+y)=y \Gamma(y)$ for the Beta function and applying the Stirling approximation $B(y) \simeq 2^{1-2 y} \sqrt{\frac{\pi}{y}}$ for large $y$, one can derive

$$
R(z) \simeq \frac{\left(z+\frac{2 s-1}{2}\right)\left(z+\frac{2 s-3}{2}\right) \ldots\left(z+\frac{1}{2}\right)}{(z+s-1)(z+s-2) \ldots(z+1)} \sqrt{\frac{\pi}{z+s}} ; \quad s=1,2,3, \ldots .
$$

Now in order to reach an action with a familiar structure let

$$
\left.R(z)\right|_{s=1} \simeq \sqrt{\pi} \sqrt{1+z}
$$


for $1+z \gg 1$. Returning Eq. (30) into the effective action (25), applying the determinant expansion $\operatorname{det}(1+y) \approx 1+\operatorname{Tr} y$ and omitting the total field strength $F$ for simplicity, an intimate result will be revealed

$$
S \approx \frac{T_{p}}{2} \sqrt{\pi} \int d^{p+1} \xi V(T) \sqrt{1-\eta^{\alpha \beta} \partial_{\alpha} T \partial_{\beta} T}
$$

This action is similar to the proposed tachyon effective action for large $T$ on an unstable $\mathrm{D} p$-brane system in [13].

\section{References}

[1] J. Polchinski, String Theory (Cambridge University Press, Cambridge, 1998).

[2] C. Johnson, D-Branes (Cambridge University Press, 2003).

[3] M. Frau, I. Pesando, S. Sciuto, A. Lerda and R. Russo, Phys. Lett. B400 (1997) 52.

[4] C.G. Callan and I.R. Klebanov, Nucl. Phys. B465 (1996) 473;C.G. Callan, C. Lovelace, C.R. Nappi and S.A. Yost, Nucl. Phys. B308 (1988) 221.

[5] P. Di Vecchia, M. Frau, I. Pesando, S. Sciuto, A. Lerda and R. Russo, Nucl. Phys. B 507 (1997) 259; P. Di Vecchia and A. Liccardo, hep-th/9912275.

[6] H. Arfaei and D. Kamani, Phys. Lett. B452 (1999) 54; F. Safarzadeh-Maleki and D. Kamani, Zh. Eksp. Theor. Phys. 146: 769, (2014); Phys. Rev. D 89, 026006,(2014).

[7] T. Asakawa, S. Sasa, S. Watamura, [hep-th/1206.6964v2]

[8] D. Kutasov, M. Marino and G. Moore, JHEP 0010 (2000) 045.

[9] P. Kraus and F. Larsen, Phys. Rev. D 63 (2001) 106004; T. Takayanagi, S. Terashima and T. Uesugi, JHEP 0103 (2001) 019.

[10] S.P. de Alwis, Phys. Lett. B 505(2001) 215; K. Okuyama, Phys. Lett. B 499 (2001) 305. T. Lee, K.S. Viswanathan and Y. Yang, J. Korean Phys. Soc. 42 (2003) 34; Z. Rezaei, Phys. Rev. D 85, 086011 (2012).

[11] F. Safarzadeh-Maleki and D. Kamani, Phys. Rev. D 90, 107902 (2014).

[12] A.A. Tseytlin, J.Math.Phys.42:2854-2871,2001; G. Arutyunov, S. Frolov, S. Theisen, JHEP 0102(2001) 002. 
[13] A. Sen, Mod. Phys. Lett. A17,1797-1804 (2002); Phys. Rev. D 68(2003)066008; M. Garousi, Nucl. Phys. B 584 (2000) 204; E. Bergshoeff, M. de Roo, J. Kluson, Phys. Rev. D 62 (2000) 126003.

[14] C. Kim, Y. Kim and C.O. Lee, JHEP 05 (2003) 020; C. Kim, Y. Kim, O-K Kwon, H-U Yee, JHEP 03, (2006) 086; C. Kim, Y. Kim, O.-K. Kwon and P. Yi, JHEP 09 (2003) 042. 